Pecvnia, 9 (2009), pp. 1-25

\title{
La cooperación como estrategia de desarrollo en redes asociativas
}

Recibido: Noviembre 2009 Aceptado: Diciembre 2009

\author{
María Cristina Acosta \\ mcacosta@econ.uba.ar \\ Griselda Verbeke \\ gverbeke@econ.uba.ar \\ Universidad de Buenos Aires
}

Fac. de Ciencias Económicas Centro de Estudios de Sociología del Trabajo Buenos Aires (Argentina)
Las acciones implementadas para realizar el principio de cooperación entre cooperativas se redefinen en un contexto que interpela, por un lado a actuar contra las consecuencias de políticas impuestas que han llevado al desempleo y pobreza extrema $y$, por otro, a plantear respuestas que comprendan una revitalización de las reglas cooperativas como un elemento central de su diferenciación en la nueva dinámica de los mercados.

Este artículo analiza dos redes agroindustriales que, con diferente grado de asociación e integración, fueron conformando estrategias que priorizan la calidad de las relaciones entre múltiples actores con intereses divergentes potenciando los resultados positivos
Actions which are implemented in order to realize the principle of cooperation among cooperatives are redefined in a context which questions, on the one hand, acting against those consequences of policies which have led to extreme unemployment and poverty and, on the other hand, suggests answers which comprehend a revitalization of cooperative rules as a central element of their differentiation in the new market dynamics.

This article discusses two industryagriculture networks which, having a different degree of association and integration, have shaped strategies which give priority to quality of relations among multiple actors with different interests, thus fostering positive results both for 
para los productores organizados en cooperativas y para la comunidad.

Palabras clave: cooperación, redes, cluster, gestión asociada. the producers organized in cooperatives and for the community.

Key words: cooperation, networks, cluster, associated management.

\section{INTRODUCCIÓN}

La cooperación entre empresas es un recurso estratégico en el mundo empresarial para responder eficazmente a un entorno competitivo. La cooperación empresarial permite, por un lado, mantener la flexibilidad de las empresas individuales al no tener que integrar todas las etapas del proceso productivo, y por otro lado, aprovechar los recursos y capacidades que poseen sus socios. Desarrollar innovación tecnológica, reducir costos y obtener economías de escala, entrar a nuevos mercados son algunos de los motivos que inducen a establecer acuerdos de cooperación.

La cooperación como construcción social no es solamente el resultado de las fuerzas del mercado, sino también una forma que ha ido progresando hacia estrategias que priorizan la calidad de las relaciones entre actores empresariales, gremiales, gobiernos locales, ONGs, e instituciones científicas, vínculos que no están mediados por las reglas del mercado (Fernández, Vigil y Seval 2008).

En este marco, para el cooperativismo, diversos factores convergen para la formación de alianzas de cooperación, entre los que se debe mencionar las expectativas para lograr una institucionalidad que permita una efectiva articulación, armonizando aspectos legislativos y productivos; la complementación de actividades productivas $\mathrm{y} / \mathrm{o}$ de servicios; y potenciar espacios comunes de participación democrática, con otras organizaciones.

Las sociedades cooperativas históricamente son el resultado de acuerdos de cooperación entre múltiples empresarios individuales que cooperan para alcanzar y mantener ventajas competitivas. Estas ventajas se expresan en el principio de la inter-cooperación cooperativa.

Las cooperativas sirven a sus socios lo más eficazmente posible y fortalecen el movimiento cooperativo trabajando conjuntamente mediante estructuras locales, nacionales, regionales e internacionales. Por lo tanto el principio de inter-cooperación ofrece dos campos de 
acción. El primero refiere al asociacionismo cooperativo, cuya finalidad es la defensa y representación de los intereses generales de las entidades asociadas, la inter-cooperación representativa, que establece formas de colaboración que respeta la autonomía decisoria de las empresas que participan en ellas. El segundo campo tiene la finalidad de potenciar su acción empresarial, conocida como inter-cooperación económica que tiende al establecimiento de vínculos entre empresas cooperativas (Alfonso Sánchez 2003).

Tradicionalmente para las empresas cooperativas se habla de las agrupaciones de segundo grado (federaciones) y de tercer grado (confederaciones), que han sido el destino natural de los procesos de integración cooperativa. Estas configuraciones son diseñadas para crear transparencia en torno a cuestiones de rendición de cuentas en el ámbito local y eficiencia central, al mismo tiempo que proveen mecanismos para que las cooperativas individuales puedan adaptarse a un entorno dinámico.

No obstante, otras formas pueden explicar las configuraciones posibles de un subsistema sectorial cooperativo que puede organizarse alrededor de clusters de actividad o redes de colaboración.

La noción de red pone el énfasis en las relaciones entre diversos actores que se integran, en los vínculos que potencian la sinergia colectiva, en la capacidad de transformación de cada parte según su relación con las demás. Una forma que están adoptando estas redes son los denominados clusters empresariales (Bakaikoa et al. 2004).

El cluster se entiende como una concentración en un mismo espacio geográfico de empresas e instituciones interconectadas en un campo particular. La proximidad entre instituciones, sean estas públicas y privadas, posibilita a sus integrantes un acceso privilegiado a relaciones estrechas entre los involucrados, una mejor información, y otras ventajas en cuanto a productividad e innovación que son difíciles de aprovechar a distancia. En la revista Ruralis (2005) se destaca que un cluster es un camino alternativo de organizar la cadena de valor, recalcando que los clusters desempeñan un papel vital en la capacidad de innovación de las empresas.

Sin embargo, ni las redes ni los cluster se construyen espontáneamente, requieren un marco de acción que asegure su misión y propicie una forma de gestión concertada en el cual adquieren relevancia, no sólo las estructuras institucionales regionales y locales impulsando a los actores sociales y económicos, sino también los actores gubernamentales 
con capacidad de desarrollar instancias de generación de políticas públicas y dirigir recursos en ese sentido (Lovering 1999).

Esto lleva a una transformación social por medio de nuevos modos de relaciones entre los actores del mercado, del Estado y de la sociedad civil. Ni completa autonomía, ni completa dependencia de uno o de los otros, sino un ajuste mutuo bajo un modelo de desarrollo entre tres (Malo 2003) que reconozca la densidad de esos entramados y las heterogeneidades en los mismos.

En este trabajo nos interesa presentar la compleja relación donde convergen diversos intereses y preferencias institucionales en dos redes agroindustriales. Teniendo en cuenta sus diferentes orígenes y características de los acuerdo, ambas constituyen el resultado de una sucesión de etapas que expresan formas de resolución de las problemáticas que afectan a pequeños productores. En cada experiencia los asociados, regidos por un acuerdo mutuo, pueden tener una participación y especialización distintas.

\section{EXPERIENCIA DE INTER-COOPERACIÓN EN EL ÁMBITO DE}

\section{LA VITIVINICULTURA}

El sector agroalimentario en las últimas décadas no ha sido ajeno a los procesos de concentración y especialización, las exigencias de competitividad en el escenario económico y la magnitud de los negocios actuales constituyen elementos necesarios para consolidar la presencia del sector a nivel regional y/o mundial que exige escala física y logística.

La vitivinicultura argentina en este contexto generó una notable innovación en el sector constituyéndose en una actividad dinámica en el país que lo ubica en el quinto lugar como productor de vino y exportador altamente competitivo, frente a los tradicionales países vitivinícolas tales como Francia, España e Italia ${ }^{1}$. Este crecimiento ha estado asociado a una fuerte inversión de capitales nacionales e internacionales y a la incorporación de tecnología en todos los eslabones de la cadena (Ruralis 2005).

\footnotetext{
Argentina está posicionada como el $8^{\circ}$ productor mundial de uva, el $5^{\circ}$ elaborador y su superficie cultivada representa el $2,6 \%$ de la superficie mundial.
} 
Por otra parte, a las innovaciones en tecnología incorporadas por el sector se añaden innovaciones en la organización de la producción, desarrollándose estrategias de ínter-cooperación a través de asociaciones diferentes en cuanto a su alcance y articulación que ha permitido orientar al sector con información, capacitación y diseño de negocios a fin de reducir las desventajas, en particular de pequeños y medianos productores, y alcanzar los estándares de calidad exigidos en los mercados internacionales.

\subsection{Caso FeCoVitA}

La vid constituye el principal cultivo de las provincias de Mendoza y San Juan ${ }^{2}$ aunque paulatinamente se ha extendido a los valles irrigados de Salta, Catamarca, La Rioja, Río Negro y Neuquén.

En la Provincia de Mendoza, como consecuencia de las sucesivas crisis que afectaron a la vitivinicultura, se fue modificando cuantitativa y cualitativamente el sector. De las 1.200 bodegas que existían en la provincia a principios de los 80, se registraban a comienzos de 2000 alrededor de 400; de ellas solamente 5 bodegas concentran el 75\% del vino común y otras 5 bodegas controlan el $40 \%$ de los vinos finos; 2 grupos económicos concentran el $70 \%$ del mercado del mosto, uno de ellos enteramente internacional y el otro, mitad extranjero y mitad nacional (Goldfarb 2007).

Sin embargo, los antecedentes del proceso de concertación de la Vitivinicultura en la Provincia se remiten a la década de los 70 . La defensa de los pequeños y medianos productores históricamente fue llevada adelante por la Asociación de Cooperativas Vitivinícolas (ACOVI). En la década de los ochenta la Asociación implementa una nueva estrategia creando la Federación de Cooperativas Vitivinícolas Argentinas (FeCoVitA) ${ }^{3}$, entidad cooperativa de segundo grado, que nucleaba originalmente a 25 cooperativas de primer grado, integradas por alrededor de 2.000 productores.

2 En Mendoza 12.400 viticultores conforman el sector proveedor de uvas con un total de 134.000 ha. En su mayoría son pequeños y medianos productores, el 55\% tiene menos de 5 ha de viñedos y el $75 \%$ hasta 10 ha. San Juan tiene el $22 \%$ del total de hectáreas cultivadas.

Cooperativa (I.N.A.C.).

3 Matrícula Nacional $n^{\circ} 9511$ otorgada por el Instituto Nacional de Acción 
El objetivo inicial de la Federación fue la defensa y representación de los intereses generales de las cooperativas y del cooperativismo cumpliendo, de esta manera, un rol fundamental en el sector vitivinicultor, y promoviendo la integración de los productores con la finalidad de potenciar su acción empresarial.

Como resultado de la estructura y de la estrategia que implementa la Federación numerosos productores individuales cooperativos con muchos años de historia en la producción vitivinícola y aún aquellos que se encontraban en situaciones ventajosas tanto económicas como productivas deciden (Neiman et al. 2006) incorporarse y articular -por vía de la coordinación- sus intereses bajo los principios y valores cooperativos participando en el proyecto asociativo.

En la actualidad la principal actividad empresarial de FeCoVitA es el fraccionamiento y comercialización de los vinos elaborados por sus 31 cooperativas asociadas ${ }^{4}$ con el aporte de unos cinco mil productores, que se agrupan en 5 Centros Regionales: Zona Norte, Zona Este, Gral. Alvear, San Rafael y Valle de Uco. En su conjunto elaboran $280.000 .000 \mathrm{Kg}$. de uva, representando cerca del $15 \%$ de la producción nacional y ubica a FeCoVitA en el quinto lugar de las empresa de bebidas de Argentina ${ }^{5}$.

\subsubsection{La trayectoria}

La cercanía y multidimensionalidad de las relaciones entre los pequeños y medianos productores y elaboradores de vinos, ha favorecido la asociación en la estructura cooperativa que reúne a los asociados bajo la figura de clientes y proveedores al mismo tiempo que propietarios, construyendo una alternativa al contexto en el que se desenvuelven y que se visualiza como pasible de transformación. Esta percepción se verifica especialmente a partir del año 1989, cuando las autoridades del gobierno de Mendoza inician el proceso de privatización de la empresa estatal Bodegas y Viñedos Giol. FeCoVitA se presenta en la licitación por la Unidad

4 Estas cooperativas limitadas son: Real del Padre, El Cerrito, Tres Porteñas, Ingeniero Giagnoni, San Carlos Sud, Algarrobo Bonito, Rama Caída, De Mendoza, Maipú, Productores de Junín, Brindis, Vista Flores, Goudge, Sierra Pintada, Viñas de Uco, Las Trincheras, Tulumaya, Norte Mendocino, Del Algarrobal, Pámp. Mendocinos, Nueva California, Norte Lavallino, Altas Cumbres, Colonia California, El Libertador, El Poniente, Tres de Mayo, Agrícola Beltrán, La Dormida, y Moluches.

5 Se ubica detrás de la Coca-Cola, Pepsi y Cervecería Quilmes. Vende 150.000.000 botellas de vino en diversos envases: tetra brick, garrafas o damajuanas y en botellas de 750 c.c. 
de Fraccionamiento y Comercialización con productores vitivinicultores pequeños y medianos de toda la provincia de Mendoza.

La privatización de $\mathrm{Giol}^{6}$ tuvo un gran impacto en la estructura económica de la provincia dada su participación en la generación de valor agregado tanto en el sector agropecuario como en el sector industrial. Adicionalmente, la privatización concretó una organización y participación diferente en el sector, la agrupación viñateros en forma cooperativa que constituía una antigua aspiración para la defensa de los precios de la uva y de los vinos mendocinos. El largo proceso de privatización que pretendía asegurar una administración más eficiente incorporando a los propios viñateros organizados en la definición del destino de la empresa, tuvo fuertes oposiciones tanto de los grandes productores, del gremialismo como del ambiente político (Fabre 2005).

Finalmente, el proyecto de reorganización de la ex empresa estatal -previamente consensuado con los dirigentes de las instituciones históricas de representación de pequeños y medianos productorescontempla acciones tendientes a promover y sostener con sus bases la necesidad de asociación y solidaridad para llevar adelante el proceso de producción.

\subsubsection{La organización}

La Federación cuando se hace cargo de la Unidad de Fraccionamiento y Comercialización, unidad de envergadura y de mayor valor estratégico y económico, se transforma en una empresa líder del mercado vitivinícola. Se adaptan las estrategias comerciales a las nuevas necesidades de la producción de vinos de calidad iniciando un periodo de crecimiento en términos económicos y financieros en un mercado muy competitivo.

En lo que se refiere a la tecnología de fraccionamiento ${ }^{7}$, La Federación ha realizado fuertes inversiones en equipos, maquinarias y

6 La bodega Giol pasó de ser un emprendimiento vitivinícola que en 1954 controlaba alrededor del $10 \%$ del proceso industrial y del $20 \%$ del comercial, a ser, veinte años después, solamente una bodega más frente al crecimiento de sus competidoras. El enorme pasivo que había acumulado explica el colapso económico de la empresa y lleva a que al Estado cumpla un nuevo rol cambiando su lógica de intervención en el mercado de la vitivinicultura.

7 En la actualidad la Federación cuenta con 9 líneas de fraccionamiento: 2 líneas de llenado rápido de 15.000 botellas/hora completamente automatizadas; 2 líneas de 4.000 botellas/hora para etiquetado autoadhesivo; 1 línea para el llenado de botellas de litro con tapa a rosca; y 4 líneas para fraccionamiento de tetra-pack. 
líneas de embotellado de última generación, de procedencias diversas tales como Alemania, Italia, Francia y Suecia.

En el plano de la representación gremial, FeCoVitA se convierte en un interlocutor significativo frente al Estado capaz de transmitir las necesidades de sus representados y también de canalizar los instrumentos para la reconversión de viñedos, incorporación tecnológica y capacitación (Neiman et al. 2006), gestiona la incorporación a mejores condiciones de acceso a los mercados y al crédito e inicia una etapa de fortalecimiento institucional.

La Federación promueve iniciativas y gestiona proyectos que tienen como objetivo actuar sobre las diversas problemáticas e ir mejorando la posición de los productores en el complejo agroindustrial (Mingo y Goldfarb 2005). Define estrategias como empresa vitivinícola en temáticas específicas (tecnología, sanidad, infraestructura), se establecen alianzas con los miembros, basada en el principio del esfuerzo propio y la ayuda mutua, para atender problemas estructurales como la competencia entre productores, la falta de información del mercado y de protección ante accidentes climáticos y escaso poder de negociación.

En cuanto a su funcionamiento, los viñateros asociados producen el grado y la cantidad del producto que las cooperativas de primer nivel necesitan para la elaboración del vino, para luego remitirla a La Federación que se encarga del envasado y su comercialización. Esta vinculación económica agrega valor a las necesidades de los asociados que atiende. Con la integración lograda los productores reciben información más específica sobre los tipos de vinos requeridos por el mercado, e invierten en la mejora de la composición varietal de los viñedos, compran maquinarias, optimizan las prácticas agrícolas y métodos de trabajo a fin de adaptar sus producciones a los requerimientos y exigencias del mercado.

Si bien la Federación fortalece la red de cooperación entre productores asociados, no es una totalidad homogénea y sin conflictos, la composición social en cuanto a capacidades y recursos de los diferentes integrantes es diversa. La necesidad de profundizar en la capacidad dialógica entre los distintos protagonistas y la puesta en común de los recursos y la información, constituye un camino para superar las tensiones que se manifiestan en la falta de integración entre los productores de una misma cooperativa, y las resistencias de muchas para incorporar tecnología de gestión para mejorar la producción, maquinaria obsoleta, entre otros. 
Las estrategias de producción y comercialización también plantean tensiones importantes entre los miembros de la Federación. En efecto, aquellos que tienen mejores condiciones productivas se orientan hacia la apertura de nuevos canales de comercialización para los vinos finos, mientras que otros consideran que esa estrategia empresarial puede limitar la producción de los asociados cuya actividad se centra en la elaboración y comercialización de vinos de baja y media gama.

En este sentido, sostener el trabajo cooperativo implica una dinámica interna particular dentro de la red y un desafío para introducir mecanismos que permitan comprometer las perspectivas e intereses de los actores, trabajar con las diferencias a fin de reducir las asimetrías y apropiaciones selectivas por parte de aquellos actores que -por estar más cerca del sistema de decisiones y/o contar con mayores recursos- obtengan beneficios de la actuación colectiva que debilite la siempre compleja cooperación inter-institucional.

En lo relativo a la conformación del gobierno, la autoridad máxima de FeCoVitA es la Asamblea General en la que se encuentran representadas las cooperativas asociadas. Los delegados que participan de la toma de decisiones en la asamblea son elegidos según los criterios pautados en el Estatuto Constitutivo de la Federación. Así, se establece que se elige un delegado por cada cincuenta asociados o fracción mayor de veinticinco, con un máximo de tres por cada cooperativa, y en proporción al monto de las operaciones en valor monetario de cada cooperativa con la Federación. También, es competencia de la Asamblea la elección de doce consejeros titulares y ocho suplentes para integrar el Consejo de Administración. Este Consejo, cuyos miembros son reelegibles es renovado anualmente por mitades y tiene por función resolver cuestiones operativas, organizativas y administrativas, siendo el encargado de nombrar a los integrantes de la Mesa Directiva, constituida por miembros del consejo, y a los gerentes que dependen de ella. Esta estructura organizativa se completa con los Consejos Regionales, constituidos por los delegados titulares de las cooperativas asociadas, que tienen por función procurar su activa participación, solucionar los problemas de distancia y ayudar a cumplir las disposiciones del Consejo de Administración en sus respectivas jurisdicciones (Fabre 2005). 


\subsubsection{La Federación vinculada}

En el marco de los procesos de apertura económica, privatización y desregulación de la economía en la década de los 90 , el sector vitivinicultor se ve fuertemente afectado llevando casi a la desaparición $^{8}$ a los pequeños productores de uva. Así se inicia para el sector un proceso de "reconversión productiva", con el objeto de promover la sustitución de cultivos orientados al vino común o "vino de mesa" por "vinos finos", valorados en el mercado mundial (Azpiazu y Basualdo 2003).

Para sostener la continuidad de las organizaciones cooperativas, la Federación -con la intención concreta de incrementar la productividad de las explotaciones en el sector elaborador, incorporar tecnología en los procesos y mejorar la calidad de los mismos- desarrolla vínculos con agencias estatales como el INTA, universidades y otras organizaciones no estatales que comparten el mismo espacio de relaciones y trabajan con el objetivo de desarrollar estrategias de intervención hacia el sector del pequeño productor vitivinícola. Algunas funcionan dentro de un área geográfica localizada mientras que otras tienen alcance territorial más amplio.

De esta forma, la Federación se ubica en un espacio intermedio en la intersección de la relación entre lo local y lo global y hace posible el flujo e intercambio de información, conocimientos y tecnologías.

En la trama de relaciones que participan los asociados de la Federación se encuentra A.C.O.V.I., precursora en el proceso de conformación y consolidación de FeCoVitA, quien asume la representación de las cooperativas vitivinícolas de la República Argentina ante los poderes públicos e instituciones privadas; gestiona las relaciones de intercambio económico y social entre las cooperativas asociadas y otras cooperativas del país y del extranjero; y asesora a sus miembros sobre cuestiones relacionadas con la organización, funcionamiento, interpretación y fines de las sociedades cooperativas.

Dentro de las organizaciones de cooperativas de productores relacionadas con el ámbito rural, la Federación mantiene con la Confederación Intercooperativa Agropecuaria Cooperativa Ltda. 
(CONINAGRO) una larga trayectoria en la representación del sector. CONINAGRO es la entidad confederada de tercer grado que representa el interés gremial, la promoción e integración del cooperativismo agrario argentino. Proporciona asistencia técnica para la resolución de problemas de comercialización, organización interna y acceso al crédito, entre otras.

La cercanía entre estos actores permitió la construcción de un espacio de referencia institucional que se sostiene en la adhesión de reglas y acciones comunes.

Desde el área de desarrollo rural en la década de los 90 se pusieron en marcha varios programas destinados a los pequeños y medianos productores vitivinicultores.

La presencia de programas públicos y la proximidad e interacción entre las instituciones de apoyo al sector y los productores, constituye una ventaja que FeCoVitA ha podido capitalizar realizando acciones conjuntas de colaboración, posibilitando a los productores una mejor competencia y control de etapas del proceso productivo.

En el convenio firmado entre el Instituto Nacional de Tecnología Agropecuaria (INTA) y FeCoVitA se asiste a los productores a través de diferentes instrumentos del Programa Federal de Apoyo al Desarrollo Rural Sustentable (PROFEDER): Programa para Productores Familiares (PROFAM) ${ }^{9}$, y Cambio Rural. Estos programas han enfatizado aspectos referidos al fortalecimiento de la organización de los productores, la transferencia tecnológica, la innovación, articulación y formación de redes con entidades del medio de carácter: local, provincial, regional y nacional, tendiendo a complementar las acciones con los diferentes proyectos regionales y especialmente al proyecto regional de Desarrollo de los Territorios.

En esta línea el INTA participa en el Plan Estratégico Vitivinícola (PEVI) junto con COVIAR (Corporación Vitivinícola Argentina) que permite la conformación conjunta de Centros de Desarrollo Vitivinícola en las provincias de Mendoza, San Juan y La Rioja.

9 Dentro del Programa Profam se desarrolla un proyecto que coordina Agencia de Extensión Rural (AER)-INTA de "Mejoramiento de la competitividad de pequeños y medianos viticultores del oasis sur del Atuel" y cuyas acciones están destinadas a productores familiares que buscan que la vitivinicultura se constituya en fuente de trabajo para la familia entera, favoreciendo el arraigo en zonas rurales, incluyendo a los hijos y mujeres en las actualizaciones tecnológicas y en el mejoramiento de la calidad de sus viñedos para poder salir al mercado con un mejor producto. 
En cuanto a las regulaciones de la actividad vitivinicultora, éstas se ejercen a través del Instituto Nacional de Vitivinicultura (INV), creado en 1959 como organismo competente para la promoción y fiscalización técnica de la producción, la elaboración y la comercialización de los productos. Adicionalmente facilita los recursos necesarios para el cumplimiento del Plan Estratégico Vitivinícola Argentina 2020 (PEVI) en el cual FeCoVitA -a través de ACOVI- participa activamente.

El PEVI, constituye un hecho sin precedentes para la Argentina ya que establece una alianza entre el sector público y privado. Elaborado en noviembre de 2006 no se centra exclusivamente en la expansión productiva o en el aumento de los volúmenes elaborados, sino en potenciar a un sector de las economías regionales con un gran valor agregado, en la organización e integración de los productores primarios; en la producción de vinos con mayor calidad constante y sostenible en el tiempo; en desarrollar el mercado latinoamericano y reimpulsar el mercado argentino de vinos; y en la fidelización de clientes y consumidores.

Los estados provinciales involucrados junto al Estado Nacional tienen un rol preponderante, ya que es el sector público -a través de los gobiernos o de organismos como el INV y el INTA- el que implementa esta iniciativa. La gestión y coordinación del plan está a cargo de la Corporación Vitivinícola Argentina (COVIAR).

La Corporación que se crea como persona jurídica de derecho público no estatal, ha mostrado un avance importante en el logro de los objetivos y la formulación de proyectos de corto y mediano alcance, claramente orientados a alcanzar las metas colectivas del sector.

El gobierno provincial también actúa en el sector, a través del Fondo para la transformación y el Crecimiento de la provincia de Mendoza, que permite a los productores agrupados a FeCoVitA acceder a las líneas de financiamiento siendo la Federación agente de retención. El productor ofrece como garantía su producción con el respaldo de la entidad.

Por otra parte, la estrecha relación con Credicoop -institución bancaria cooperativa- hace referencia a la importancia que tienen las sociedades cooperativas de trabajar conjuntamente en el plano de intereses comunes. El Banco tiene un acuerdo con FeCoVitA para el acceso de sus asociados a un programa de créditos para cosecha y acarreo de la producción. 
La Federación en la articulación de los diferentes niveles que coordina -productores, cooperativas, técnico- ha generando acciones colectivas con el objetivo de actuar sobre la problemática de los pequeños y medianos productores que facilite las condiciones político-institucionales para el desarrollo económico y social de los asociados y sus comunidades. Estas acciones requieren esfuerzos de capacitación, aprendizajes colectivos, adhesión de los protagonistas y acompañamiento inter-institucional en el territorio. La creación de nuevos vínculos y relaciones que trabajen de manera sinérgica, han contribuido a que se potencien las capacidades de los grupos y comunidades indispensables para resolver las necesidades y llevar adelante un proceso de manera sostenida y a mayor escala.

Pensar en una estrategia socioeconómica territorial supone la presencia de organizaciones públicas propiciando el aprendizaje permanente y generando plataformas de servicios técnicos, crediticios y científicos, que movilice y acompañe los recursos y capacidades presentes en el territorio.

En el mismo sentido, el INTA encaró en el NOA un proceso innovador que reúne diversos actores alrededor de un proyecto participativo para recuperar la capacidad efectiva de crear alternativas para mejorar la calidad de vida de pequeños productores apícolas.

\section{EXPERIENCIA DE INTER-COOPERACIÓN EN EL ÁMBITO DE} LA APICULTURA

La cuestión del desarrollo rural se convirtió en un punto focal en el noroeste argentino donde las condiciones de pobreza se profundizaron. Atender esta problemática fue el objetivo de numerosos programas y proyectos gubernamentales nacionales y provinciales orientados a apoyar a los pequeños y medianos productores agropecuarios. En particular, nos interesa la conformación de un modelo de intervención orientado al desarrollo económico del sector apícola.

Argentina es el tercer productor mundial de miel después de China y Estados Unidos, representando el 6\% del total mundial (SAGPyA 2009). Se trata de una actividad, secundaria o complementaria, llevada a cabo en todo el país por unos 33.000 apicultores que cuentan con aproximadamente 4.000 .000 colmenas, según datos del RENAPA (Registro Nacional de Productores Apícolas), que coordina y administra la Dirección 
Nacional de Alimentos. La comercialización se realiza a través de unas 100 empresas, de las cuales 10 concentran el $70 \%$ de las exportaciones. Pese a liderar el mercado mundial de miel, la Argentina se ha caracterizado por ofrecer un producto sin ningún grado de diferenciación, destinado a segmentos del mercado de bajos precios relativos. Históricamente, los canales de comercialización convergieron en unas pocas empresas exportadoras y los apicultores no fueron estimulados para la obtención de un producto de calidad (Caporgno 2005).

La provincia de Buenos Aires concentra más del $50 \%$ de la producción nacional de miel (Fundación export.ar 2007), no obstante la apicultura se ha extendido y existen actualmente desarrollos en varias provincias del país. En el Noroeste y Centro la actividad presenta una atomización de la producción, sin presencia de experiencias asociativas destacables, asimetrías entre exportadores y apicultores, y escasa aplicación de protocolos de calidad lo que ha sido una barrera adicional para mejorar la productividad y competitividad requerida, tanto en los estándares de los mercados nacionales como internacionales, y a la venta de la producción con reducido grado de diferenciación (PROAPI-INTA 2008).

No obstante, la apicultura es una actividad con bajas barreras de entrada donde existen amplias posibilidades de expansión para los pequeños emprendedores.

En este contexto se llevaron a cabo una serie de acciones estratégicas en redes de cooperación que dieron origen al Cluster Apícola que se sostiene en la innovación y el intercambio de conocimientos cumpliendo un rol central en el crecimiento y desarrollo económico.

El modelo de intervención utilizado para la reactivación del sector, parte de la participación y consolidación de una red interinstitucional con la participación activa de los pequeños y medianos productores, organismos técnicos, universidades, gobiernos locales, y organizaciones sociales. De esta forma, la red se convierte en el medio a través del cual circula la información y el conocimiento. Así, a mayor densidad de redes mayor será el capital social, y mayor la capacidad de innovación (Lundvall 2002) y mejora de la eficacia del sistema.

Desde esta perspectiva se formula el Proyecto Integrado de Desarrollo Apícola (PROAPI) en el ámbito público para el desarrollo de acciones estratégicas, que permiten incrementar beneficios sectoriales, tanto en lo productivo como en lo comercial, otorgando prioridad a la innovación tecnológica que facilita el tránsito a condiciones competitivas 
dinámicas. De este modo, se intentaba revertir las dificultades de las micro y pequeñas empresas para competir y superar el desafío de la sustentabilidad empresarial (Bedascarrasbure 2005).

La estrategia planteada por el proyecto comienza con la asistencia de grupos técnicos (GAT) que organizan a los pequeños apicultores del NOA y centro del país, continúa en la integración de los pequeños productores a empresas jurídicamente constituidas, y de allí al cluster apícola. Esta estrategia se concreta en el año 2002 cuando se incuba la Cooperativa Norte Grande Ltda. en el Parque de Innovación Tecnológica del INTA en Famaillá ${ }^{10}$, Provincia de Tucumán. Las incubadoras de empresas son herramientas utilizadas para apoyar el surgimiento de nuevos emprendimientos, en especial, de base tecnológica o con fuerte contenido innovador ${ }^{11}$.

El desarrollo de nuevas empresas incubadas está relacionado con el interés regional de generar nuevos puestos de trabajo y mejores condiciones de producción desde la actuación preponderante de agentes gubernamentales. Concentran sus esfuerzos en el fortalecimiento de los emprendedores y sus proyectos, a través de la prestación de diversos servicios durante el transcurso de una o varias de las etapas que demanda el proceso de formación de empresas ${ }^{12}$.

Con esta orientación y en el marco del Acuerdo INTA-PROAPIIPACYM $^{13}$, se inicia el proceso de integrar a la Cooperativa Norte Grande ${ }^{14}$ a los pequeños apicultores. Originalmente la cooperativa contaba con diez asociados, la mayoría eran microemprendedores que se iniciaron en los grupos de Cambio Rural.

10 La estructura del INTA en la Provincia de Tucumán está integrada por la sede del Centro Regional Tucumán - Santiago del Estero, el Campo Experimental Leales (CER) y la estación Experimental Agropecuaria Famaillá (EEA) con sus diez Unidades de Extensión dependientes.

11 El Estado Nacional interviene con políticas de promoción de incubadoras y parques tecnológicos: FONTAR (Fondo Tecnológico Argentino), los Aportes No Reembolsables para Programas de Incubadoras de Empresa de Base Tecnológica de la Agencia Nacional de Promoción Científica y Tecnológica (ANPCyT), Programa Especial de Incubadoras de Empresas, Parques y Polos Tecnológicos de la Secretaría de Ciencia y Tecnología (Fardelli y Ciancio 2006).

12 Las incubadoras pueden ser clasificadas según objetivo institucional en Incubadoras para el fomento de la innovación y en Incubadoras para el desarrollo de nuevas empresas (EIMS). El EIMS (European Innovation Monitoring System) identifica dos modelos básicos, dependiendo del foco y el grado de compromiso institucional que los agentes tienen con el establecimiento de incubadoras.

13 IPACYM: Instituto Provincial de Acción Cooperativa y Mutual de Tucumán.

14 La cooperativa obtiene su matrícula nacional $N^{\circ} 23420$ en febrero de 2002. 
Posteriormente, en diciembre de 2003, la cooperativa incorpora nuevos socios, pequeños apicultores de Tucumán, Salta, Jujuy y Santiago del Estero hasta totalizar en la actualidad 95 asociados ${ }^{15}$. En conjunto agrupan 4.700 colmenas que representan el 6,3\% del total de colmenas de la región y el 3,8 del total de apicultores, ofreciendo productos como material vivo certificado primicia, mieles, propóleos, polen, jalea real y cera.

La equidad, el compromiso, la confianza mutua y la honradez integran el código de principios y valores que orienta las acciones de sus asociados, y son la base de aprendizajes colectivos de normas, comportamientos y reglas aprobadas por consentimiento. El cumplimiento de los valores ha logrado que las actividades de la Cooperativa Norte Grande fueran declaradas de interés por la Honorable Cámara de Diputados de la Nación en septiembre de 2004, como modelo asociativo de pequeños apicultores. Al mismo tiempo la aplicación de estrictos protocolos de producción, desarrollados por el INTA para asegurar la calidad de los productos, y la trazabilidad desde el apiario respetando el medio ambiente, y el compromiso asumido por todos los asociados con los valores mencionados han logrado la certificación de FLO (Fair Trade Label Organization) para comercializar en Europa a través de Mercado Justo. De esta forma la red amplió las posibilidades para acciones estratégicamente articuladas e impulsar el Cluster Apícola del NOA.

\subsection{Caso Cluster Apícola}

El Cluster Apícola, es una red pública-privada creada en el 2002 con capacidad para realizar investigación y desarrollo abierto a todas las empresas vinculadas al sector, y facilitar su competencia en los segmentos de productos de alta calidad.

Como objetivos se propone: fomentar la producción, generar un sistema de gestión de la calidad y de capacitación que permita desarrollar productos certificados a nivel internacional, crear instrumentos financieros para la expansión del sector, y promocionar los productos de calidad certificada obtenidos.

15 Según datos consignados en la página institucional ApiNet (http://www.apinetla.com.ar/NG/servicio_desarrollo.htm). 
El alcance territorial del cluster abarca las provincias de Jujuy, Salta, Tucumán, Santiago del Estero y Santa Fe. Actualmente, sobre unas 43.150 colmenas, su facturación es cercana a los 2 millones de dólares (Alba et al. 2008).

\subsubsection{La organización}

El proceso de su formación ha constituido un "territorio" en el sentido en que lo define Camagni (2002), es decir, un espacio caracterizado por la existencia de elementos clave: un sistema de externalidades tecnológicas localizadas, un sistema de relaciones sociales y económicas (capital relacional), y un sistema de gobernanza local formado por un conjunto de actores privados y administraciones públicas locales.

La densidad institucional y organizativa de la red está integrada por más de 50 entidades, entre ellas siete organismos gubernamentales de distintos niveles que han apoyado activamente este proceso $^{16}$.

En el sector productivo el cluster está compuesto por 233 apicultores, organizados en 18 empresas. Las empresas que adoptan diferentes condiciones jurídicas están reunidas alrededor de dos núcleos productivos, uno en el NOA, con la Cooperativa Norte Grande, y otro en la región Centro, con la cooperativa $\operatorname{COSAR}^{17}$ creada en el año 1999 en el marco del Programa Apícola Provincial que se propone promover la formación de Asociaciones Departamentales de Apicultores. Actualmente COSAR formada por 10 grupos de apicultores de Santa Fe y Córdoba está integrada por un total de 110 productores.

En cuanto a los aspectos institucionales, en el cluster participan cuatro universidades nacionales (Tucumán, Salta, Jujuy, y del Centro de la Provincia de Buenos Aires) y el INTA-PROAPI que dio origen al

16 Ministerio de Desarrollo Social de la Nación (MDS), Consejo Federal de Inversiones (CFI), Ministerio de Ciencia, Tecnología e Innovación productiva de la Nación (MCTIP), Instituto Provincial de Acción Cooperativa y Mutual de la Provincia de Tucumán (IPACYM), Secretaría de Innovación y Desarrollo Tecnológico de Tucumán (SIDETEC), Ministerio de la Producción de la provincia de Jujuy (MPJ), Consejo de la Microempresa de Jujuy (CMJ).

17 Desde el Programa Cambio Rural de la SAGPyA y con el apoyo tecnológico del Proyecto Integrado de Desarrollo Apícola del INTA (PROAPI) se formaron 6 grupos apícolas para trabajar en conjunto en la calidad de miel para exportar. Los grupos se transformaron en empresas jurídicamente constituidas, realizaron inversiones en salas de extracción de miel bajo normas higiénico-sanitarias que cumplen con las condiciones de calidad. Consolidado este proceso se conformó la Cooperativa COSAR Ltda., en la que cada grupo es un socio (Alba et al. 2008). 
agrupamiento. Investigación y desarrollo constituye el aspecto relevante y distintivo del cluster, es el nodo de transferencia tecnológica y sede de muchos de los ensayos de experimentación adaptativa, desarrollando una serie de tecnologías que permiten obtener un producto de calidad certificada, producción sin antibiótico y mayores rendimientos (Alba et al. 2008).

Con el objetivo de fortalecer la transferencia de tecnología a los apicultores y la capacitación de los mismos, el cluster creó en el 2002 una Red integrada por 20 escuelas localizadas en zonas del NOA postergadas social y económicamente. Ellas establecen sus propios objetivos y los planes de capacitación son consensuados con los actores locales teniendo en cuenta las demandas y necesidades específicas de cada territorio.

La red de relaciones con otros actores del entorno socioinstitucional supone el rol activo de los agentes locales en el proceso de desarrollo local, y el fortalecimiento continuo de su participación en espacios de toma de decisiones. Es decir, para canalizar las iniciativas propuestas es necesario que el espacio interinstitucional así construido facilite la concertación entre los actores imprescindibles para el desarrollo. Esto significa definir puntos cruciales de acuerdo entre las diferentes lógicas e intereses de los participantes, más allá de las racionalidades de cada uno (Arocena 2001).

\subsubsection{El gobierno}

El conjunto de actores empresas, instituciones científicas y tecnológicas, y entes gubernamentales están representados en la Asociación denominada Conglomerado Competitivo de la Industria Apícola Zona NOACentro. El directorio de la asociación está formado por 11 miembros: 5 representan a las empresas, 3 a las instituciones científico-tecnológicas, y 3 a los organismos de promoción. Tiene a su cargo llevar adelante la ejecución del proyecto Consolidación y Desarrollo Social y Competitivo del Aglomerado Apícola del Noroeste-Centro Argentino, en el marco de los Proyectos Integrados en Aglomerados Productivos (PITEC). Este instrumento, se implementa desde la Agencia Nacional de Promoción Científico y Tecnológica (ANPCyT), dependiente del Ministerio de Ciencia, Tecnología e Innovación Productiva de la Nación (MCTIP) y financiado por el Fondo Tecnológico Argentino (FONTAR). 
El proyecto propone expandir e incorporar al cluster a los pequeños apicultores del Noreste Argentino (NEA), incrementar la expansión horizontal, eliminar la brecha tecnológica hacia el interior del conglomerado, y lograr que el $100 \%$ de la producción se realice bajo sistemas de gestión de calidad.

Para ello está trabajando sobre la construcción de una red de laboratorios y observatorios de mercado con las Universidades de Jujuy y Tucumán, con el INTA, con la Red de laboratorios del cluster; y en la conformación de un Departamento Técnico para Conserjerías Tecnológicas.

Por otro lado, la estructura del cluster se completa con un Sistema de gestión de la calidad, estructura organizacional que deriva de la innovación en la implementación del paquete tecnológico. En este Sistema están representados los productores, las empresas que posean salas de extracción, los asesores técnicos y el Organismo Gestor de la Calidad. Este último conformado por los apicultores que integran las cooperativas, y sus funciones son: a) la toma de decisiones respecto de los negocios a realizar, b) controlar el normal funcionamiento de todo el sistema, y c) realizar los controles y penalizaciones a las empresas o productores que no cumplan con las normas establecidas. Además, es el responsable de habilitar el ingreso de nuevos grupos al sistema, teniendo en cuenta que los mismos deben demostrar previamente estar en condiciones de implementar los protocolos (Alba et al. 2008).

La experiencia del cluster apícola, aunque reciente, ha alcanzado un grado elevado de organización en la conformación de una red regional en condiciones de sustentar la expansión horizontal y vertical de la actividad a nivel de pequeños apicultores asociados.

Las fortalezas del cluster se basan en la tecnología dura desarrollada, y los valores sobre los que se sustenta, constituyendo una importante fuente de competitividad ya que reduce los costos de transacción entre los actores que intervienen (Acosta y Verbeke 2007).

Para ello la interacción del sector productivo, el sector científico y tecnológico, y el institucional de fomento ha contribuido a generar un espacio representativo de los intereses negociados del conjunto de los actores.

Finalmente, el equipo interinstitucional e interdisciplinario conformado para resolver los problemas más urgentes en términos de gestión de la calidad e incorporación de innovación tecnológica, ha 
producido una sinergia donde lo que uno hace se realimenta con lo que hace el otro generando así una importante masa crítica que es capaz de colocar al sector a la vanguardia de la apicultura mundial.

\section{CONSIDERACIONES FINALES}

Las exigencias de competitividad en el sistema agroalimentario argentino plantean la necesidad de la integración de los productores en sistemas de cooperación que den respuesta a las exigencias actuales de magnitud, volumen y calidad del mercado internacional. Esto interpela a una estrategia de conformación de redes en la que participan grupos de individuos o colectivos, en instancias de concertación institucional sostenida en el tiempo y articulada entre sí para actuar en un área determinada.

Hemos presentado dos experiencias de ínter-cooperación con diferentes grados de asociación, integradas por unidades de producción que orientaron estratégicamente a los sectores vitivinicultor y apícola.

En primer lugar se presenta a Bodegas FeCoVitA Coop. Ltda, institución cooperativa de segundo grado fundada para la defensa de los intereses político-gremial de los pequeños y medianos productores de Mendoza, y su acción empresarial, a través de la cual realiza la distribución y comercialización complementariamente a la difusión de información, y capacitación de sus miembros. En segundo lugar, el cluster Apícola del Noroeste y Centro Argentino, asociación ad hoc públicaprivada centrada en la investigación y desarrollo de tecnologías que favorezca una producción de miel de calidad antes que el volumen de los emprendimientos apícolas.

Ambas experiencias, que representan formas estructurales alternativas aún con características históricas y culturales particulares, fortalecieron las capacidades locales, y consolidaron una red institucional. El desarrollo de innovaciones tecnológicas y organizacionales respaldadas por diversas instituciones -públicas y privadas, lucrativas y sin fines de lucro, organismos de promoción y de política pública- abrieron una vía a la formación y permanencia de empresas individuales que organizaron sus actividades con valores tales como la confianza y el compromiso, ejes directrices de la red y de la reconversión productiva de los pequeños productores. 
Con una lógica territorial-horizontal las redes han movilizado a un conjunto de actores en torno a un problema considerado crucial para la actividad y la comunidad local. Esta lógica establece consensos sobre los temas de interés común a fin de alcanzar los objetivos propuestos, de modo que las diferencias entre actores con racionalidades específicas sean articuladas de manera tal que ninguna sea dominante en el tratamiento de las problemáticas y resulten en beneficios para el conjunto.

Asimismo, las dos experiencias presentan diferencias en cuanto a sus orígenes, conformación y modos de intervención en el desarrollo rural.

La Federación, iniciativa de los propios productores, es una organización formalizada y consolidada en el tiempo por cooperativas agropecuarias locales definidas geográficamente y unidas por lazos de proximidad, de necesidad y con un proyecto común. La estructura horizontal es propia de una organización cooperativa donde las reglas democráticas -más allá de la diversidad de opiniones existentes-, la igualdad de condiciones de los asociados, la primacía del trabajo sobre el capital, y la responsabilidad compartida constituyen sus principios rectores.

Esta Federación cuenta con una larga e importante trayectoria, en sus orígenes reunió a productores, algunos de los cuales contaban con experiencia en trabajo cooperativo. El proceso de fortalecimiento institucional, a partir de la privatización de la empresa GIOL, presenta para los pequeños y medianos vitivinicultores nuevos desafíos al demandar habilidades y conocimientos industriales y comerciales inéditos. No obstante, en esta etapa los productores, aprovechando las ventajas de adherir a un sistema cooperativo federado, logran consolidar una importante presencia en el mercado vitivinícola, situación improbable de alcanzar para las cooperativas por separado y para los pequeños productores independientes.

En la medida en que la Federación complejiza sus actividades, y crecen sus interacciones con un contexto institucional más amplio, intercambia mayor cantidad y calidad de recursos materiales y tecnológicos, información y conocimiento que son utilizados estratégicamente por la entidad para alcanzar visibilidad y sostenibilidad.

En cambio, el cluster tiene una estructura ad-hoc recientemente conformada que reúne empresas territoriales, organismos del Estado, centros científicos y tecnológicos, y surge en el marco de los programas de desarrollo rural como forma de precipitar una instancia de 
concertación territorial que facilite la comunicación y difusión de innovaciones, factor clave para el desarrollo socio económico. Las relaciones que establecen los miembros involucrados con las redes productivas tecnológicas y la red de entidades de formación tienden, a mediano y largo plazo, a promover la asociatividad, la cooperación y la planificación participativa.

Entonces, estas estructuras viejas y nuevas, surgieron impulsadas por las demandas de los actores locales y fueron incrementando la densidad del tejido social local, esencial para el desarrollo local. La fluidez de la comunicación y la participación efectiva de los asociados asegura la consecución de los objetivos, dado que las partes se encuentran enlazadas de forma tal de generar un circuito que, si falla en algún punto, se compromete la totalidad. Es por ello que esta forma de organización conlleva el control mutuo entre cada una de las partes garantizando así que la cadena no se corte.

La valorización de los procedimientos y el impacto en la resolución de los problemas en la red depende del grado de integración e inter-cooperación de los actores involucrados. Una red productiva integrada permite alcances importantes al combinar innovaciones tecnológicas y organizacionales, y logra que la red funcione como un sistema único que se sostiene por la adaptación voluntaria de las relaciones cercanas e integradas. La red, de esta manera, trabaja en un nivel más elevado por la existencia de vínculos establecidos de esta manera.

Las herramientas e instrumentos gestados por políticas públicas nacionales y provinciales han contribuido significativamente en la formación y gestión de estas estructuras. En el caso del cluster, el Proyecto Integrado de Aglomerados Productivos (PITEC) implementado desde la Agencia Nacional de Promoción Científica y Tecnológica, ha sido dinamizador, ya que pudieron acceder a todas las líneas de apoyo necesarias para afianzar la organización, y comenzar el proceso de planificación estratégica del mismo. En el caso de la vitivinicultura, el sector propició una alianza entre el sector público y privado que, como resultado, logra la concertación de los actores del sector en el Plan Estratégico Vitivinícola Argentina 2020 para potenciar las economías regionales con un gran valor agregado a través de la organización e integración de los productores primarios. En esta iniciativa FeCoVitA, junto a las entidades representativas del sector cooperativo en la región, forma parte del Plan con importantes implicaciones en la economía y en los aprendizajes colectivos para sus miembros y de la región. 
Más allá de las diferencias entre las distintas experiencias expuestas, ambas coinciden en la necesidad de generar actividades económicas que permitan la sustentabilidad del proyecto asociativo que los integra, potenciar las capacidades y la creatividad de los productores, para construir un espacio de desarrollo que resulte beneficioso para los actores y la comunidad.

BIBLIOGRAFÍA

Acosta, M.C. y G. VerbeKe (2007) "Los alcances de la participación en las asociaciones con pluriactividad". Trabajo presentado en el $V$ Simposio de Análisis Organizacional "El campo Organizacional y las nuevas fronteras de lo público y lo privado". Buenos Aires, Centro de Estudios de Sociología del Trabajo. Facultad de Ciencias Económicas. Universidad de Buenos Aires.

Alba, M.; E. Bedascarrasbure, J. García y C. Álvarez (2008) "Innovación y desarrollo social en el medio rural argentino: El caso del Cluster Apícola del Noroeste y Centro Argentino". Trabajo presentado en el VII CIER "Cultura, Innovación y Territorio".

Alfonso SÁnCHEZ, R. (2003) "Posibilidades y Regulación de los Procesos de Integración en España. Cooperativas de segundo grado, grupos cooperativos, fusiones". R. CHAVES, G. FAJARDO y R. NAMORADO (coords.) Integración empresarial cooperativa: posibilidades, ventajas $e$ inconvenientes. Valencia: Ed. Ciriec-España.

ARoCENA, J. (2001) "Centralización y Deslocalización: La Búsqueda De Alternativas". Ponencia presentada en el VI Congreso Internacional del CLAD sobre la Reforma del Estado y de la Administración Pública, Buenos Aires, CLAD.

ASPIAZU, D. y E. BASUALDO (2003) Las exportaciones vitivinícolas durante la postconvertibilidad. Buenos Aires: Facultad Latinoamericana de Ciencias Sociales (FLACSO).

BakaikoA, B.; A. Begiristain, A. ERraste y G. GolkoetXea (2004) "Redes en innovación cooperativa", Revista de Economía Pública, Social y Cooperativa, 49, pp. 263-294.

Bedascarrasbure, E. (2005) "La paradoja de la Miel", Anales del Congreso Apícola de MERCOSUR. Uruguay: Punta del Este. 
CAMAGNI, R. (2002) "On the Concept of Territorial Competitiveness: Sound or Misleading", Urban Studies, 39, n³9, pp. 2395-2411.

CAPORGNo, J. (2005) "Miel Argentina de Calidad Diferenciada". Ponencia presentada en el Primer congreso apícola del norte argentino. Salta [http://www.culturaapicola.com.ar/apuntes/congresos/1\%20congreso \%20noroeste/06_javier_caporgno.pdf].

FABRE, P. (2005) La privatización de Bodegas y Viñedos Giol. Una experiencia exitosa. Buenos Aires: Naciones Unidas. Comisión Económica para América Latina (CEPAL), Documento LC/BUE/R.263.

FARDELLI CORROPOLESE, C. y M. CIANCIO (2006) Entornos Institucionales para la creación y desarrollo de Empresas: las incubadoras de empresas. Buenos Aires: Universidad de General Sarmiento.

FernánDEZ, V.R.; J.I. VIGIL y M. SeVAL (2008) "Clusters y Cadenas de Valor ¿Instrumentos de Desarrollo Económico en América Latina?", Ponencia presentada en las II Jornadas Nacionales de Investigadores de las Economías Regionales, IX Encuentro de la Red de Economías Regionales en el Marco del Plan Fénix Tandil, Provincia de Buenos Aires [http://www.econ.uba.ar/planfenix/aportes/comisionc.htm].

FUNDACIÓN EXPORT.AR (2007) Plan de Promoción Sectorial Apícola. Buenos Aires [http://www.minagri.gob.ar/SAGPyA/economias_regionales/_api cultura/_publicaciones/_publicaciones/plan_promocion_sectorial.pdf].

GOLDFARB, L. (2007) "Reestructuración productiva en el sector vitivinícola mendocino. La construcción social de un paradigma de calidad", Ponencia presentada en el II Seminario Internacional. Nuevos Desafíos del Desarrollo en América Latina. La perspectiva de jóvenes académicos. Córdoba: Universidad Nacional de Río Cuarto.

LOVERING, J. (1999) "Theory Led by Policy. The Inadequacies of the New Regionalism", International Journal of Urban and Regional Research, 23, pp. 379-395.

LUNDVALL, B. (2002) "Estados-Nación, capital social y desarrollo económico. Un enfoque sistémico de la creación de conocimiento y el aprendizaje en la economía global", Revista de Economía Mundial, 7, pp. 69-90.

MALO, M.C. (2003) "La Cooperación y la Economía Social". M. VuotTo (comp.) Economía Social Precisiones Conceptuales y Algunas Experiencias Históricas. Buenos Aires: Ed. Altamira.

MINGO, E. y L. GoldFARB (2005) "Reestructuración productiva y nuevas formas de representación de los pequeños productores vitivinícolas. El caso de la Federación de Cooperativas Vitivinícolas Argentinas", Trabajo presentado en las IV Jornadas Interdisciplinarias de Estudios Agrarios. 
Buenos Aires, CIEA. Facultad de Ciencias Económicas. Universidad de Buenos Aires.

Neiman, G.; M. Berger, S. Arroñade, F. Fabio, L. Goldfarb, A. Karol, E. MINGO y M. NeIMAN (2006) "Diversidad de las formas de representación de intereses entre organizaciones de pequeños productores del agro argentino: base social, reivindicaciones y articulaciones". M. MANZANAL, G. NEIMAN y M. LATTUADA (compiladores) Desarrollo rural, organizaciones $e$ instituciones. Buenos Aires: Ediciones CICCUS.

PORTER, M. (1998) "Clusters and the new economics of competition", Harvard Business Review, 6, Nov/Dec, pp. 77-90.

PROAPI-INTA (Programa Nacional Agropecuario, Instituto Nacional de Tecnología Agropecuaria) (2008) Programa Nacional Apícola Documento Base. Buenos Aires [http://www.inta.gov.ar/info/cadena/miel/PNAPI DocbaseJulio08.pdf.

RURALIS (2005) (Especial Vitivinícola) 7, Año II, octubre, noviembre y diciembre, pp. 1-40.

SAGPyA (Secretaria de Agricultura, Ganadería, Pesca y Alimentos. Dirección Nacional de Agroindustria. Área Apícola) (2009). Introducción al sector apícola argentino [http://www.minagri.gob.ar/SAGPyA/ec onomias_regionales/_apicultura/_publicaciones/_informes/sector_apic ola_argentino.pdf]. 